\title{
Blockade of Endogenous Anterior Hypothalamic Atrial Natriuretic Peptide with Monoclonal Antibody Lowers Blood Pressure in Spontaneously Hypertensive Rats
}

\author{
Ren-Hui Yang, * Hongkui Jin,* Yiu-Fai Chen, ${ }^{*}$ J. Michael Wyss, ${ }^{*}$ and Suzanne Oparil* \\ *Hypertension Program, Division of Cardiovascular Diseases, Department of Medicine and the Department of Cell Biology \\ and Anatomy, University of Alabama at Birmingham, Birmingham, Alabama 35294
}

\begin{abstract}
We have previously shown that the atrial natriuretic peptide (ANP) content of the anterior hypothalamic region of $\mathrm{NaCl}-$ sensitive spontaneously hypertensive rats (SHR-S) is higher than that of Wistar-Kyoto (WKY) rats. ANP has been shown to inhibit neuronal norepinephrine release and to reduce the excitability of hypothalamic neurons. This study tested the hypothesis that blockade of endogenous ANP in the anterior hypothalamus by local microinjection of a monoclonal antibody to ANP (MAb KY-ANP-II) lowers blood pressure in SHR-S. Purified MAb KY-ANP-II (0.055 and 0.55 $\mu$ g) or control mouse IgG in $200 \mathrm{nl}$ saline was microinjected into the anterior hypothalamic area (AHA) of conscious SHR-S and control WKY rats. As a further control, Mab KY-ANP-II $(0.55 \mu \mathrm{g})$ was microinjected into the posterior hypothalamic area (PHA) of SHR-S. Anterior hypothalamic microinjection of MAb KY-ANP-II caused significant dose-related decreases in mean arterial pressure (MAP) and heart rate (HR) in SHR-S but not in WKY rats. Control injections of equal volumes of IgG had no effect on MAP or HR. Microinjection of Mab KY-ANP-II into PHA produced no significant alteration in MAP or HR in SHR-S. These data provide the first demonstration that endogenous ANP in a region of brain known to influence cardiovascular function mediates BP and HR control in the rat. These findings suggest that the increased endogenous ANP in the anterior hypothalamus of SHR-S may be involved in the central regulation of BP in this model. ( $\mathrm{J}$. Clin. Invest. 1990. 86:1985-1990.) Key words: monoclonal antibody to ANP • microinjection • central nervous system • brain • hypertension
\end{abstract}

\section{Introduction}

Atrial natriuretic peptide (ANP), ${ }^{1}$ the vasodilator/natriuretic peptide originally described in mammalian atria, is also found

Address correspondence and reprint requests to Dr. Suzanne Oparil, 1034 Zeigler Research Building, University of Alabama at Birmingham, UAB Station, Birmingham, AL 35294.

Received for publication 1 May 1990 and in revised form 10 July 1990.

1. Abbreviations used in this paper: AHA, PHA, VHA, anterior, posterior, and ventral hypothalamic peptide; ANOVA, analysis of variance; ANP, atrial natriuretic peptide; $h$, heart; HR, heart rate; MAP, mean arterial pressure; NE, norepinephrine; SHR-S, spontaneously hypertensive rats-Okamato strain; WKY, Wistar-Kyoto.

J. Clin. Invest.

(C) The American Society for Clinical Investigation, Inc.

0021-9738/90/12/1985/06 \$2.00

Volume 86, December 1990, 1985-1990 in the brain (1-7). Immunoreactive ANP has been detected in various brain regions by radioimmunoassay of extracted tissue and by immunocytochemistry $(2,4,7-9)$. ANP mRNA and ANP receptors have also been described, indicating that an intact ANP synthetic, processing, and effector system is present in rat brain $(1-6,10)$. Immunocytochemical data indicate that ANP-containing cell bodies are present in highest density in the anterior hypothalamic region adjacent to the anteroventral tip of the third ventricle $(4,9)$. This region is involved in the development and maintenance of experimental hypertension and in fluid and electrolyte balance $(4,9)$.

Previous studies from our laboratory have shown that the ANP content of the anterior hypothalamic region of the saltsensitive spontaneously hypertensive rat of the Okamoto strain (SHR-S) is significantly higher than that of the WistarKyoto (WKY) rat (11). ANP content of the other brain regions examined, including posterior hypothalamic area (PHA), ventral hypothalamic area (VHA), pons, and medulla, did not differ between strains. The anterior hypothalamic region contains neurons that have a depressor effect when excited by electrical stimulation or noradrenergic input (12-15). ANP has been shown to inhibit norepinephrine (NE) release from peripheral nerve terminals (16-18) and from an adrenal pheochromocytoma (PC 12) cell line in culture (19) and to reduce the excitability of rat hypothalamic neurons in vivo (20). We hypothesized that the increased ANP in the anterior hypothalamus of SHR-S may produce local tonic inhibition of $\mathrm{NE}$ release, thus reducing excitation of depressor neurons and elevating BP. Blockade of this ANP effect by administering anti-ANP antibody or an ANP B receptor blocker would stimulate $N E$ release, thus exciting depressor neurons in anterior hypothalamic area (AHA) and lowering BP.

This study tested the hypothesis that blockade of endogenous ANP in AHA by local microinjection of a monoclonal antibody to ANP (MAb KY-ANP-II) lowers BP in SHR-S. We used as controls, microinjections of IgG purified from mouse ascites fluid into AHA and microinjections of Mab KY-ANPII into PHA. Our results demonstrated that microinjection of a blocking monoclonal antibody to ANP into the AHA produced significant decreases in both BP and heart rate (HR) in SHR-S but not in WKY rats. Neither microinjection of IgG into AHA nor of MAb KY-ANP-II into PHA altered BP. These results suggest that increased endogenous ANP in AHA may be involved in central regulation of BP in SHR-S.

\section{Methods}

SHR-S and normotensive WKY control rats were obtained from Taconic Farms (IBU-3 colony; Germantown, NY) at 9 wk of age. All rats were maintained four per cage at constant humidity $(60 \pm 5 \%)$, temperature $\left(24 \pm 1^{\circ} \mathrm{C}\right)$, and light cycle ( $0600-1,800$ hours). All rats were provided a standard rat diet (5001; Ralston-Purina, Richmond, IN) and 
free access to food and water. For 1 wk before acute study, rats were acclimatized to these diet and housing conditions.

$2 \mathrm{~d}$ before the acute experiment, each rat was anesthetized with sodium pentobarbital $(50 \mathrm{mg} / \mathrm{kg}$ i.p.) and a catheter (polyethylene PC-10 fused with PE-50) was implanted into the abdominal aorta through the right femoral artery. The rat was then placed into a stereotaxic apparatus, the skin overlying the midline of the skull was incised, and a small hole was drilled through the appropriate portion of the skull. A guide cannula (26-gauge stainless steel tubing) was lowered to a position $1.0 \mathrm{~mm}$ dorsal to the intended site of microinjection, according to the coordinates of Paxinos and Watson (21) (anteroposterior [AP], $-1.6 \mathrm{~mm}$ from bregma; mediolateral [ML], 0.65; and dorsoventral [DV], 8.8), and fixed to the skull with dental acrylic. A 32-gauge obturator (stainless steel wire) was inserted into the guide cannula after implantation.

$48 \mathrm{~h}$ after surgery, the arterial catheter was connected to a model CP-01 pressure transducer (Century Technology Company, Inglewood, CA) coupled to a polygraph (Model 7; Grass Instruments, Quincy, MA). Mean arterial pressure (MAP) and HR were measured simultaneously. After a 40-min stabilization period, the obturator was removed from the guide cannula and replaced with an inner cannula (32-gauge stainless steel tubing) filled with the agent to be administered. The tip of the inner cannula extended $1 \mathrm{~mm}$ beyond the guide cannula. The inner cannula was attached to a $0.5-\mu \mathrm{l}$ Hamilton syringe through tubing (polyethylene PE-20) filled with saline. A small air bubble was made between the saline and the injection solution. Rats were randomly injected with two doses $(0.055$ and $0.55 \mu \mathrm{g})$ of MAb KY-ANP-II, which had been purified by the procedure outlined below, or mouse IgG $(0.55 \mu \mathrm{g})$ purified from ascites fluid as a control in $200 \mathrm{nl}$ saline. The first injection was administered as soon as the rat's vital signs returned to baseline (2-5 min). MAP and HR were allowed to return to baseline before subsequent injection. The interval between injections was 60-90 min in all animals. All microinjection experiments were carried out in conscious, free moving rats.

As an additional control, in a separate series of experiments, Mab KY-ANP-II $(0.55 \mu \mathrm{g})$ was microinjected into PHA, an area in which ANP content and NE release are not different in SHR-S and WKY rats $(11,22)$. Surgery, arterial cannulation, and brain cannula implantation were performed as above except that the coordinates of Paxinos and Watson were AP, $3.6 \mathrm{~mm}$ from bregma; $\mathrm{ML}, 0.4$; and DV, 8.3.

At the conclusion of the experiments, $1 \%$ methylene blue solution $(200 \mathrm{nl})$ was administered into AHA or PHA through the cannula. The rat was then killed by decapitation without prior anesthesia and the cannula was removed from the brain. The brain was removed from the skull and sectioned at $30 \mu \mathrm{m}$ on a freezing microtome (Slee Medical Equipment Ltd., London, UK). Sections were mounted and stained with $1 \%$ thionin for verification of the microinjection site.

The monoclonal antibody used in these studies was the high affinity antibody against rat $\alpha$-ANP, the 28-amino acid form of ANP, produced by Mukoyama, et al., and named MAb KY-ANP-II (23). MAb KY-ANP-II recognizes human ( $\alpha$-h ANP) and rat ANP ( $\alpha$-r ANP) equally and blocks the ability of both exogenous and endogenous ANP to elevate plasma cyclic GMP levels (24). IgG containing MAb KY-ANP-II was purified from mouse ascites fluid $(1 \mathrm{ml})$ using a protein A agarose column (25). Retained IgG with MAb KY-ANP-II was eluted from the protein $\mathrm{A}$ column with $3 \mathrm{M} \mathrm{MgCl}_{2}$ and dialyzed against $0.9 \%$ saline overnight. Dialysate was then lyophilized and reconstituted to its original volume $(1 \mathrm{ml})$ with $0.9 \%$ saline. The purified IgG $(1.1 \mathrm{mg} / \mathrm{ml})$ with MAb KY-ANP-II bound $50 \%$ of $\mathrm{I}^{125}$-ANP $(17,000 \mathrm{cpm})$ at $1: 100,000$ final dilution in a total volume of $500 \mu 1$ (26). The two doses of MAb KY-ANP-II ( 0.055 and $0.55 \mu \mathrm{g})$, which were used in the current experiment, are equivalent to the anti-ANP antibody contained in 0.055 and $0.55 \mu \mathrm{l}$ of mouse ascites fluid. This is $\sim 0.05$ and $0.5 \%$ of the peripheral intravenous dose $(100 \mu \mathrm{l}$ of ascites fluid) of this monoclonal antibody used in previous studies by Itoh et al. (24).

Statistical analysis. Results are expressed as means \pm SEM. Twoway analysis of variance (ANOVA) (strain $\times$ time, and dose $\times$ time) was performed to assess the differences in MAP and HR responses to MAb KY-ANP-II between doses and between strains. One-way ANOVA was used to compare differences over time in each SHR or WKY group. Significant differences were then subjected to NewmanKeuls post hoc analysis. $P<0.05$ was considered significant.

\section{Results}

15 SHR-S and $10 \mathrm{WKY}$ rats were studied. Histological examination confirmed that cannulae were properly placed in the anterior and central portions of AHA as defined by Saper et al. (27) in 8 SHR-S and 9 WKY rats and in PHA in 5 SHR-S rats. In 1 SHR-S, the cannula entered the sagittal sinus; in a second SHR-S, the cannula was in the third ventricle. In 1 WKY, cannula placement was in the dorsal hypothalamic area. These 3 rats were excluded from the analysis of experimental results.

SHR-S had significantly higher basal MAP and lower body weight (BW) than WKY rats at the time of study (Table I). There was no difference in basal HR between SHR-S and WKY (Table I). Microinjection of MAb KY-ANP-II into the AHA resulted in significant dose-related depressor and bradycardic responses in SHR-S $(P<0.01$, two way ANOVA) but not in WKY rats (Fig. 1). Both MAP and HR responses to MAb KY-ANP-II began almost immediately after injection, reached maximal levels at 5-10 min, and returned to baseline 30 min postinjection. MAP and HR responses to both doses of MAb KY-ANP-II were significantly greater in SHR-S than in WKY for the first 30 min postinjection $(P<0.05$ for the lower dose; $P<0.01$ for the higher dose, two way ANOVA) (Fig. 1). Maximal high dose MAb KY-ANP-II-induced changes in MAP and HR in SHR-S were $-17.5 \pm 1.1 \mathrm{mmHg}$ and $-42.5 \pm 5.1 \mathrm{bpm}$, respectively (Fig. 2). Microinjection of $\mathrm{IgG}$ and saline vehicle into AHA did not alter MAP or HR significantly in either strain (Fig. 3). Microinjection of MAb KYANP-II into the PHA did not cause significant alterations in MAP or HR in SHR-S (Fig. 4).

\section{Discussion}

This study demonstrated that microinjection of MAb KYANP-II into AHA caused significant dose-related decreases in MAP and HR in SHR-S but not in WKY rats. Control injections of equal volumes of IgG into AHA had no effect on MAP or HR. Further, injection of MAb KY-ANP-II into the PHA of SHR-S did not significantly alter MAP or HR. Taken together with the findings that anterior hypothalamic stores of endogenous ANP are increased selectively in SHR-S compared with WKY controls, and that ANP inhibits neuronal NE release, these data suggest that endogenous ANP in AHA may be involved in the centrally mediated regulation of BP in SHR.

Table I. Basal Levels of $B W, M A P$, and $H R$

\begin{tabular}{lccc}
\hline & BW & \multicolumn{1}{c}{ MAP } & HR \\
\hline & \multicolumn{1}{c}{$g$} & \multicolumn{1}{c}{$m m H g$} & $b p m$ \\
WKY, AHA $(n=9)$ & $266.8 \pm 2.4$ & $116.7 \pm 2.9$ & $399.4 \pm 8.8$ \\
SHR, AHA $(n=8)$ & $225.8 \pm 2.0^{*}$ & $167.2 \pm 1.9^{*}$ & $396.3 \pm 15.4$ \\
SHR, PHA $(n=5)$ & $229.6 \pm 3.1^{*}$ & $170.0 \pm 6.1^{*}$ & $378.0 \pm 8.6$
\end{tabular}

${ }^{*} P<0.01$, compared with WKY. 

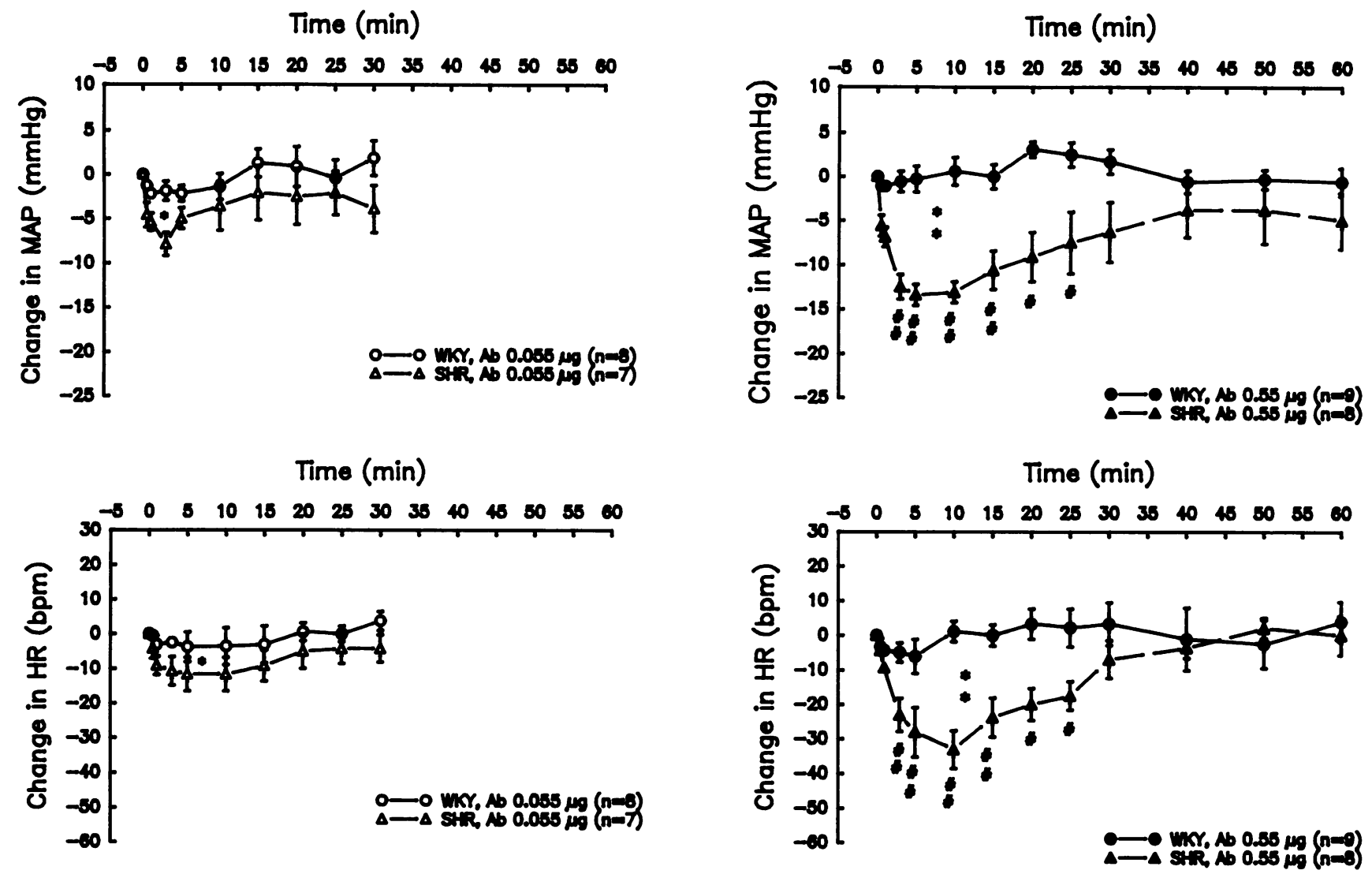

Figure 1. Effects of anterior hypothalamic microinjection of monoclonal antibody $(A b)$ to ANP on MAP, and HR in SHR and WKY rats. ${ }^{*} P$ $<0.05, P<0.01$, comparison between SHR and WKY at first $30 \mathrm{~min}$ (two-way ANOVA); ${ }^{*} P<0.05, P<0.01$ compared with time 0 .

Several lines of evidence indicate that brain ANP is altered in SHR compared with normotensive control rats. Studies from a number of laboratories have demonstrated that the ANP content of the hypothalamus, pons, and septum is signifcantly elevated in SHR compared with age-matched WKY controls (28-33). The ANP content of whole hypothalamus in young prehypertensive SHR is not significantly different from WKY, but hypothalamic ANP decreases with age in WKY and not in SHR, reaching significantly different levels by $8 \mathrm{wk}$ of age, at which time BP in SHR is significantly greater than in WKY controls (30). Our previous studies demonstrated that ANP stores are significantly elevated in the anterior hypothalamic region, but not in the posterior hypothalamic region, ventral hypothalamic region, pons, or medulla of 9-wk-old SHR-S compared with WKY (11). Intravenous injection of
ANP into the intact rat, or application of ANP to brain slices in vitro, causes marked increases in cyclic GMP levels in hypothalamus and brain stem of SHR that are significantly greater than those in WKY controls $(31,34)$. These observations suggest that ANP-sensitive particulate guanylate cyclase activity in the hypothalamus and brain stem is greater in SHR than in WKY rats $(31,34)$. The functional significance of these alterations in endogenous brain ANP and its second messenger with respect to cardiovascular regulation has not been tested.

A mechanism by which endogenous ANP in the AHA might participate in $\mathrm{BP}$ control is via regulation of NE release by AHA nerve terminals. Abundant evidence has shown that both endogenous and exogenous NE act in AHA to lower BP and HR via action on $\alpha_{2}$-adrenergic receptors. Chemical (6hydroxydopamine) destruction of the ventral noradrenergic

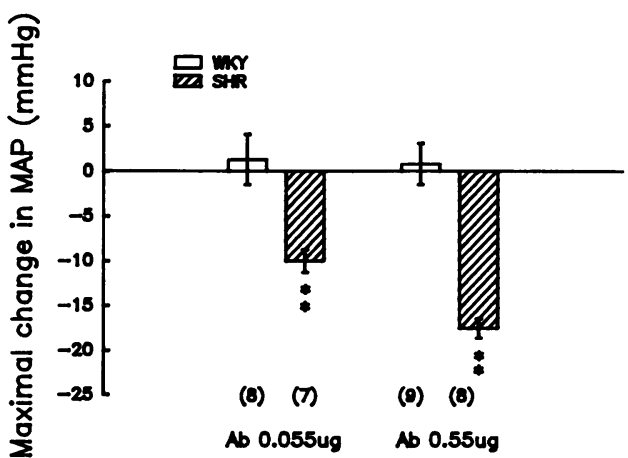

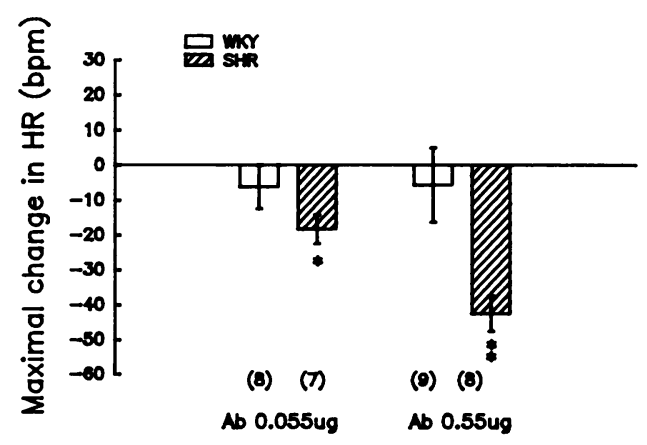

Figure 2. Maximal effects of anterior hypothalamic microinjection of monoclonal antibody $(A b)$ to ANP on MAP and HR in SHR and WKY rats. ${ }^{*} P$ $<0.05, P<0.01$, compared with WKY. 

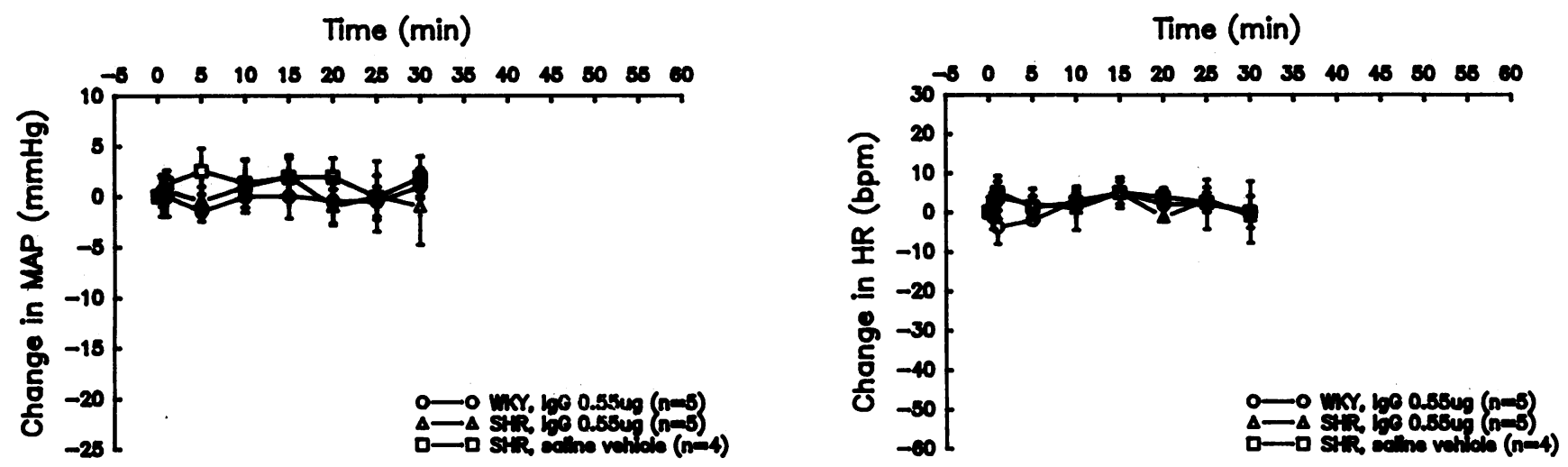

Figure 3. Effects of anterior hypothalamic microinjection of IgG and saline vehicle on MAP and HR in SHR and WKY rats.

pathway of normotensive Wistar rats selectively depletes NE stores in the anterior hypothalamus and increases BP and HR (15). Further, electrical stimulation of the anterior hypothalamus reduces BP and $\mathrm{HR}$ in both normotensive and hypertensive rats (14). Microinjection of NE or the $\alpha_{2}$-adrenergic agonists clonidine and guanabenz into AHA lowers BP and HR in the intact, conscious rat (13). This effect is exaggerated in SHR-S, particularly after dietary $\mathrm{NaCl}$ supplementation, compared with normotensive controls. Receptor binding studies using $\left[{ }^{3} \mathrm{H}\right]$ para amino clonidine have shown that $\alpha_{2}$-adrenoreceptor numbers in anterior hypothalamus of SHR-S are significantly elevated compared with WKY (36). This observation, combined with the finding that the NE content of the anterior hypothalamic region is higher in SHR-S than in WKY (22), presumably reflects upregulation of $\alpha_{2}$-adrenoreceptors in anterior hypothalamus of SHR-S in response to reduced local NE release. Reductions in endogenous NE activity in the AHA would be expected to decrease inhibition of sympathetic outflow and thereby cause BP to rise. ANP has been shown to reduce neuronal excitability in the rat hypothalamus (20). When locally applied to individual neurons, ANP inhibits the neuronal firing rate (20). Further, ANP inhibits NE release from peripheral sympathetic nerve terminals (16-18) and from PC 12 cells in culture (19). In this study, therefore, it would be expected that locally microinjected MAb KY-ANPII would block the action of anterior hypothalamic ANP, reducing NE release and/or excitability of neurons in this brain area, thereby decreasing MAP and HR. This effect would be expected to be enhanced in SHR-S compared with WKY because of the higher levels of ANP found in anterior hypothalamus of the hypertensive strain. The observation that microinjection of MAb KY-ANP-II into AHA of WKY rats had no effect on MAP suggests that NE release in AHA of normotensive WKY rats is not under tonic inhibitory control by ANP.

The MAb KY-ANP-II used in this study was purified from mouse ascitic fluid produced by Mukoyama et al. (23). Mab $\mathrm{KY}$-ANP-II is a high affinity monoclonal antibody directed towards the $\mathrm{NH}_{2}$ terminus of $\alpha$-ANP. This antibody binds equally to $\alpha$-rANP and $\alpha$-hANP. Intravenous injection of MAb KY-ANP-II significantly suppresses the increase in plasma cyclic GMP levels after administration of exogenous $\alpha$-hANP or administration of AVP with secondary stimulation of ANP release from the heart (24). Further, elevated plasma cyclic GMP levels in SHR of the stroke prone substrain (SHR-SP) and DOCA-salt rats were significantly reduced by intravenous administration of MAb KY-ANP-II, indicating that MAb KY-ANP-II can block the biological activity of $\alpha$ rANP in the intact rat. We demonstrated that purified MAb KY-ANP-II bound $50 \%$ of ${ }^{125}$ I-ANP at $1: 100,000$ final dilution, confirming the efficacy of the antibody.

Previous studies of the effects of central administration of exogenous ANP on MAP and HR in SHR and normotensive control rats have yielded inconsistent results, mainly because of differences in species of peptide, site, dose, and schedule of administration employed. Administration of human ANP ( $\alpha$ human ANP or human $\mathrm{ANP}_{1-25}$ ) into lateral ventricle did not alter MAP or HR in SHR or normotensive Wistar or WKY control rats (37-39), whereas injection of $\alpha$-rat ANP ( $\alpha$-rANP, $0.2-0.8 \mu \mathrm{g}$ ) significantly increased MAP and HR in conscious SHR and WKY rats. The pressor and tachycardic responses to $\alpha$-rANP were greater in SHR than in WKY controls (37). In contrast, injection of atriopeptin III ( $\left.\mathrm{rANP}_{5-28} ; 20 \mu \mathrm{g} / \mathrm{kg}\right)$ into the lateral ventricle did not alter MAP (40), while injection of the same dose of atriopeptin III into the fourth ventricle
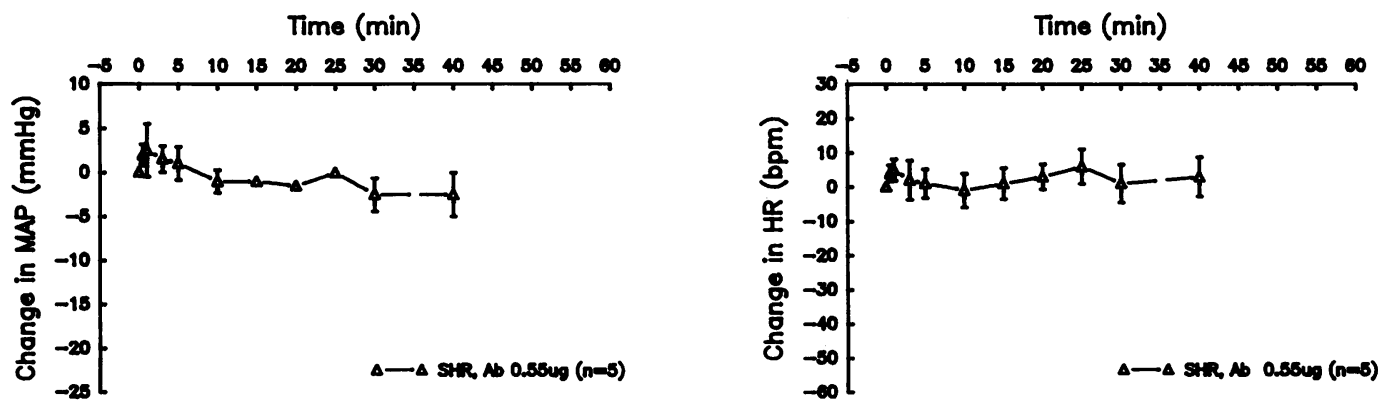

Figure 4. Effects of posterior hypothalamic microinjection of monoclonal antibody $(A b)$ to ANP on MAP and HR in SHR. 
caused a significant decrease in MAP but no change in HR in conscious SHR and WKY rats (41), suggesting that the site of depressor action of ANP was in hindbrain. Microinjection of rat ANP ( (ANP $_{5-27} ; 10^{-7} \mathrm{M}$ ) into nucleus tractus solitari, cuneate nucleus, or the spinal trigeminal complex produced slight but significant decreases in MAP and HR in anesthetized Wistar rats $(42,43)$. No responses were found when the same dose of the rANP was injected into the medial longitudinal fasciculus, hypoglossal nucleus, area postrema, or dorsal motor nucleus of the vagus (42). In contrast, microinjection of atriopeptin III ( $\left.\mathrm{rANP}_{5-28} ; 20-40 \mathrm{pmol}\right)$ into the preoptic suprachiasmatic nucleus produced significant increases in MAP and HR in Sprague-Dawley rats (44). Taken together, these data suggest that central injection of exogenous rat ANP but not human ANP affects MAP and HR in rats, and that the site for ANP depressor action is in hindbrain, especially in nucleus tractus solitari, whereas the preoptic suprachiasmatic nucleus is the site for ANP pressor action. The cardiovascular effects of localized microinjection of ANP into AHA have not been examined in either normotensive or hypertensive rats. Katsuura et al. have reported that lateral ventricular injection of antiANP antiserum, which was prepared by immunizing rabbits with $\alpha$-ANP-(17-28), potentiated the dipsogenic response to water deprivation and angiotensin II injection in rats (45). These investigators did not assess MAP or HR responses to the antiserum.

In summary, this study demonstrated that acute blockade of endogenous anterior hypothalamic ANP with a high affinity monoclonal antibody to ANP lowered MAP and HR in SHR-S. This is the first demonstration that endogenous ANP in a region of brain known to influence cardiovascular function mediates BP and HR control in the rat. These data suggest that augmented endogenous ANP in the anterior hypothalamus plays an role in BP regulation in SHR-S.

\section{Acknowledgments}

The authors thank Dawn MacEldowney and April Sandlin for typing the manuscript. We also express our appreciation to Dr. Hiroo Imura (Kyoto University) for his generous gift of KY-ANP-II antibody.

This work was supported in part by research grants from the National Institutes of Health, National Heart, Lung, and Blood Institute, HL-22544, HL-07457, and HL-35051, and by the Council for Tobacco Research, USA, Inc.

\section{References}

1. Tanaka, I., K. S. Misono, and T. Inagami. 1984. Atrial natriuretic factor in rat hypothalamus, atria and plasma: determination by specific radioimmunoassay. Biochem. Biophys. Res. Commun. 124:663-668.

2. Quirion, R., M. Dalpe, A. D. Lean, J. Gutkowska, M. Cantin, and J. Genest. 1984. Atrial natriuretic factor (ANF) binding sites in brain and related structures. Peptides. 5:1167-1172.

3. Kawata, K., K. Nakan, N. Morii, Y. Kiso, H. Yamashita, H. Imura, and Y. Sano. 1985. Atrial natriuretic peptide: topographical distribution in the rat brain by radioimmunoassay and immunohistochemistry. Neuroscience. 16:521-546.

4. Saper, C. B., D. G. Standaert, M. G. Currie, D. Schwartz, D. M. Geller, and P. Needleman. 1985. Atriopeptin immunoreactive neurons in the brain: presence in cardiovascular regulatory areas. Science (Wash. DC). 27:1047-1049.
5. Gardner, D. G., G. P. Vlasuk, J. D. Baxter, J. C. Fiddes, and J. A. Lewicki. 1987. Identification of atrial natriuretic factor gene transcripts in the central nervous systems of rat. Proc. Natl. Acad. Sci. USA. 84:2175-2179.

6. Standaert, D. G., P. Needleman, M. L. Day, R. Weigand, and J. E. Krause. 1988. Expression of the gene for preproatriopeptin in the central nervous system of the rat. Brain. Res. 464:7-13.

7. Jacobowitz, D. M., G. Skofitsch, H. R. Keiser, R. L. Eskay, and N. Zamir. 1985. Evidence for the existence of atrial natriuretic factorcontaining neurons in the rat brain. Neuroendocrinology. 40:92-94.

8. Standaert, D. G., P. Needleman, and C. B. Saper. 1986. Organization of atriopeptin-like immunoreactive neurons in the central nervous system of the rat. J. Comp. Neurol. 253:315-341.

9. Gibson, T. R., G. M. Wildey, S. Manaker, and C. C. Glembotski. 1986. Autoradiographic localization and characterization of atrial natriuretic peptide binding sites in the rat central nervous system and adrenal gland. J. Neurosci. 6:2004-2011.

10. Inagami, T., I. Tanaka, J. C. McKenzie, M. Nakamaru, R. Takayanagi, T. Imada, R. Pochet, A. Resibois, M. Naruse, K. Naruse, and T. Shibasaki. 1989. Discovery of atrial natriuretic factor in the brain: its characterization and cardiovascular implication. Cell. $\mathrm{Mol}$. Neurobiol. 9:75-85.

11. Jin, H., Y. F. Chen, R. H. Yang, Qc. Meng, and S. Oparil. 1988. Impaired release of atrial natriuretic factor in $\mathrm{NaCl}$ loaded spontaneously hypertensive rats. Hypertension. 11:739-744.

12. Sawchenko, P. E., and L. W. Swanson. 1982. The organization of noradrenergic pathways from the brainstem to the paraventricular and supraoptic nuclei in the rat. Brain. Res. Rev. 4:275-325.

13. Wyss, J. M., R. H. Yang, H. Jin, and S. Oparil. 1988. Hypothalamic microinjection of alpha $a_{2}$-adrenoceptor agonists causes greater sympathoinhibition in spontaneously hypertensive rats on high Nacl diets. J. Hypertens. 6:805-813.

14. Miyajima, E., and R. D. Bunag. 1984. Chronic cerebroventricular infusion of hypertonic sodium chloride in rats reduces hypothalamic sympatho-inhibition and elevates BP. Circ. Res. 54:566-575.

15. Benarroch, E. E., M. S. Balda, S. Finkielman, and V. E. Nahmod. 1983. Neurogenic hypertension after depletion of norepinephrine in anterior hypothalamus induced by 6-hydroxydopamine administration into the ventral pons: role of serotonin. Neuropharmacology. 22:29-34.

16. Kuchel, O., W. Debinski, K. Pacz, N. T. Buu, R. Garcia, J. R. Cussion, P. Larochelle, M. Cantin, and J. Genest. 1987. An emerging relationship between peripheral sympathetic nervous activity and atrial natriruretic factor. Life Sci. 40:1545-1551.

17. Kakamaru, M., and T. Inagami. 1986. Atrial natriuretic factor inhibits norepinephrine release evoked by sympathetic nerve stimulation in isolated perfused rat mesenteric arteries. Eur. J. Pharmacol. 123:459-461.

18. Drewett, J. G., G. J. Trachte, and G. R. Marchand. 1988. Atrial natriuretic factor inhibits adrenergic and purinergic neurotransmission in the rabbit isolated vas deferens. J. Pharmacol. Exp. Ther. 248:135142.

19. Drewett, J., G. Marchand, R. Zeigler, and G. Trachte. 1988. Atrial natriuretic factor inhibits norepinephrine release in an adrenergic clonal cell line (PC12). Eur. J. Pharmacol. 150:175-179.

20. Wong, M., W. K. Samson, C. A. Dudley, and R. Moss. 1986. Direct, neuronal action of atrial natriuretic factor in the rat brain. Neuroendocrinology. 44:49-53.

21. Paxinos, G., and C. Watson. 1986. The rat brain in stereotaxic coordinates. Academic Press, New York.

22. Chen, Y. F., Q. Meng, J. M. Wyss, H. Jin, and S. Oparil. 1988. High $\mathrm{NaCl}$ diet reduces hypothalamic norepinephrine turnover in hypertensive rats. Hypertension. 11:55-62.

23. Mukoyama, M., K. Nakao, T. Yamada, H. Itoh, A. Sugawara, Y. Satio, H. Arai, K. Hosoda, G. Shirakami, N. Morri, S. Shiono, and H. Imura. 1988. A monoclonal antibody against $\mathrm{N}$-terminus of $\alpha$-atrial natriuretic polypeptide ( $\alpha$-ANP): a useful tool for preferential detec- 
tion of naturally circulating ANP. Biochem. Biophys. Res. Commun. 151:1277-1284.

24. Itoh, H., K. Nakao, M. Mukoyama, T. Yamada, K. Hosoda, G. Shirakami, N. Morii, A. Sugawara, Y. Saito, S. Shiono, H. Arai, I. Yoshida, and H. Imura. 1989. Chronic blockage of endogenous atrial natriuretic polypeptide (ANP) by monoclonal antibody against ANP accelerates the development of hypertension in spontaneously hypertensive and deoxycorticosterone acetate-salt-hypertensive rats. J. Clin. Invest. 84:145-154.

25. Lindmark, R., K. Thoren-Tolling, and J. Sjoquist. 1983. Binding of immunoglobulins of protein A and immunoglobulin levels in mammalian sera. J. Immunol. Methods. 62:1-13.

26. Chen, Y. F., H. Jin, R. Paul, and S. Nagahama. 1988. Blunted pressor responsiveness to quinpirole, a specific dopamine $D_{2}$ receptor agonist, in conscious deoxycorticosterone acetate $/ \mathrm{NaCl}$ hypertensive rats is related to atrial natriuretic peptide release. J. Pharmacol. Exp. Ther. 246:485-492.

27. Saper, C. B., L. W. Swanson, and W. M. Cowan. 1978. The efferent connections of the anterior hypothalamic area of the rat, cat and monkey. J. Comp. Neurol. 182:575-600.

28. Ruskoaho, H., and J. Leppaluoto. 1988. Immunoreactive atrial natriuretic peptide in ventricles, atria, hypothalamus, and plasma of genetically hypertensive rats. Circ. Res. 62:384-394.

29. Kuchel, O., W. Debinski, G. Thibault, M. Cantin, and J. Genest. 1988. Ganglionic, spinal cord and hypothalamic atrial natriuretic factor: its distribution, origin and possible role in spontaneously hypertensive rats. J. Hypertens. 6(Suppl):279-281.

30. Imada, T., R. Takayanagi, and T. Inagami. 1985. Changes in the content of atrial natriuretic factor with the progression of hypertension in spontaneously hypertensive rats. Biochem. Biophys. Res. Commun. 133:759-765.

31. Takayanagi, R., T. Imada, R. T. Grammer, K. S. Misono, M. Naruse, and T. Inagami. 1986. Atrial natriuretic factor in spontaneously hypertensive rats: concentration changes with the progression of hypertension and elevated formation of cyclic GMP. J. Hypertens. 4(Suppl):303-307.

32. Morri, N., N. Kawauwa, H. Itoh, A. Sugawara, M. Sakamoto, T. Yamada, S. Shiono, M. Kihera, M. Mano, Y. Yamori, and H. Imura. 1988. Increased tissue level of atrial natriuretic polypeptide in the hypothalamus and septum of spontaneously hypertensive rats. $J$. Hypertens. 4(Suppl):309-312.

33. Debinski, W., O. Kuchel, N. T. Buu, and G. Thibault. 1988.
Atrial natriuretic factor in the spinal cord of normotensive and hypertensive rats. Peptides. 9:1101-1105.

34. Takayanagi, R., R. T. Grammer, and T. Inagama. 1986. Regional increase of cyclic GMP by atrial natriuretic factor in rat brain: markedly elevated response in spontaneously hypertensive rats. Life Sci. 39:573-580.

35. Folkow, B., B. Johannson, and B. Oberg. 1959. A hypothalamic structure with a marked inhibitory effect on tonic sympathetic activity. Acta Physiol. Scand. 262-270.

36. Klangkalya, B., W. Sripairojthikoon, S. Oparil, and J. M. Wyss. 1989. High $\mathrm{NaCl}$ diets increase anterior hypothalamic $\alpha 2$ adrenoceptors in SHR. Brain Res. 451:77-84.

37. Squadrito, F., N. Frisina, M. Buemi, R. Sturniolo, A. Autolitano, V. Magri, G. Squadrito, and A. P. Caputi. 1989. A comparison of synthetic human and rat ANP administered intracerebroventricularly in freely moving normotensive and hypertensive rats. J. Cardiovasc. Pharmacol. 13(Suppl):527-530.

38. Casto, R., J. Hilbig, G. Schroeder, and G. Stock. 1987. Atrial natriuretic factor inhibits central angiotensin II pressor responses. $H y$ pertension. 9:473-477.

39. Shimizu, T., G. Katsuura, M. Nakamura, K. Nakao, N. Morii, Y. Itoh, S. Shiono, and H. Imura. 1986. Effect of intracerebroventricular atrial natriuretic polypeptide on BP and urine production in rats. Life Sci. 39:1263-1270.

40. Levin, E. R., S. Mills, and M. A. Weber. 1989. Central nervous system mediated vasodepressor action of atrial natriuretic factor. Life Sci. 44:1617-1624.

41. Levin, E. R., M. A. Weber, and S. Mills. 1988. Atrial natriuretic factor-induced vasodepressin occurs through central nervous system. Am. J. Physiol. 255 (Heart Circ. Physiol. 24):H616-H622.

42. McKitrick, D. J., and F. R. Calaresu. 1988. Cardiovascular responses to microinjection of ANF into dorsal medulla of rats. Am. J. Physiol. 255:R182-187.

43. Ermirio, R., P. Ruggeri, C. E. Cogo, C. Molinari, and F. R. Calaresu. 1989. Neuronal and cardiovascular responses to ANF microinjected into the solitary nucleus. Am. J. Physiol. 256:R577-R582.

44. Sills, M. A., K. Q. Nguyen, and D. M. Jacobowitz. 1985. Increases in HR and BP produced by microinjections of atrial natriuretic factor into the AV3V region of rat brain. Peptides. 6:1037-1042.

45. Katsuura, G., M. Nakamura, K. Inouye, M. Kono, K. Nakao, and H. Imura. 1986. Regulatory role of atrial natriuretic polypeptide in water drinking in rats. Eur. J. Pharmacol. 121:285-287. 